\title{
Phase Changes in $f$-electron Metals: Discrepancies Between Experiment and Theory
}

\author{
J. Akella \\ S.T. Weir \\ G.S. Smith \\ C. Ruddle \\ This paper was prepared for submittal to the \\ International Conference on Condensed Matter under High Pressures \\ Bombay, India \\ November 11-15, 1996
}

October 1996

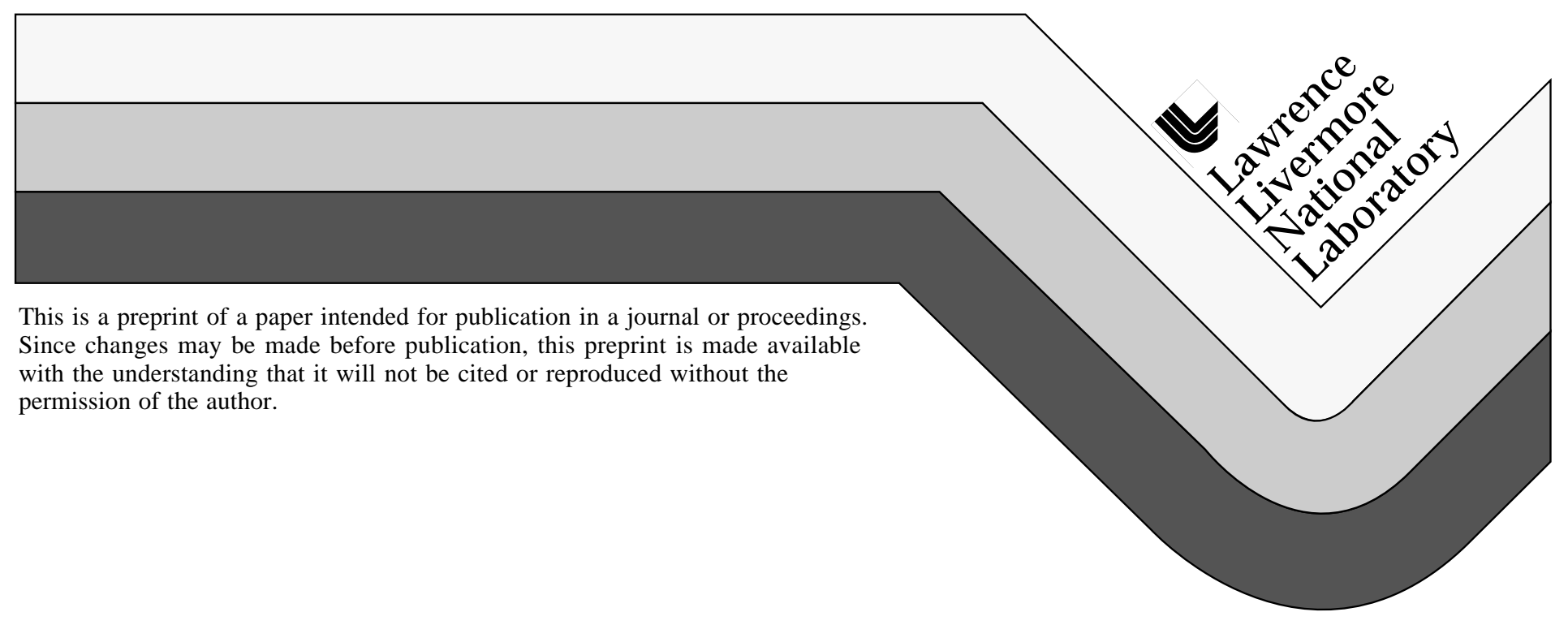




\section{DISCLAIMER}

This document was prepared as an account of work sponsored by an agency of the United States Government. Neither the United States Government nor the University of California nor any of their employees, makes any warranty, express or implied, or assumes any legal liability or responsibility for the accuracy, completeness, or usefulness of any information, apparatus, product, or process

disclosed, or represents that its use would not infringe privately owned rights. Reference herein to any specific commercial product, process, or service by trade name, trademark, manufacturer, or otherwise, does not necessarily constitute or imply its endorsement, recommendation, or favoring by the United States Government or the University of California. The views and opinions of authors expressed herein do not necessarily state or reflect those of the United States Government or the University of California, and shall not be used for advertising or product endorsement purposes. 


\title{
Phase changes in $f$-electron metals: Discrepancies between experiment and theory
}

\author{
Jagannadham Akella, Samuel T. Weir, and Chantel Ruddle \\ Lawrence Livermore National Laboratory \\ Livermore, CA 94550
}

\begin{abstract}
$\underline{\text { Abstract }}$
Using a diamond-anvil cell (DAC) phase transformation and room temperature Equation of State (EOS) for some lanthanides and actinides were studied to multimegabar (megabar $=100$ $\mathrm{GPa}$ ) pressures. Experimental data are compared with the theoretically predicted crystal structural changes and the pressure-volume relationships.

A generalized trend for the phase transformations in the lanthanides can be seen, which has broad agreement with theory. There is a general agreement between theory and experiment for the structural changes in the lighter actinides, however in detail there are some discrepancies still.

We conclude that an accurate and robust theoretical base for predicting the phase transformations in the $f$-electron metals can be developed by incorporating the DAC data as markers for fine tuning the theory.
\end{abstract}

\section{Introduction:}

Understanding the systematics in the high pressure behavior of lanthanides and actinides is important to developing appropriate theory that can predict phase changes in $f$-electron metals even up to multimegabar pressures. Equations of state and phase transformations for lanthanides and actinides at room temperature were studied experimentally to multimegabar pressures (megabar = $100 \mathrm{GPa}$ ) using diamond-anvil cells (DAC). For the past several years with the availability of advanced computational tools, theoretical investigation has intensified for predicting the crystallographic phase transformations in $f$-electron metals.

Even though in a broader sense both experimental observations and theoretical predictions are in general agreement, in detail, however, there are some discrepancies. These differences are manifested more in some of the light actinide metals which have low symmetry structures at ambient conditions and are also investigated to relatively high pressures.

The purpose of this paper is to examine different conclusions arrived at for some metals from the experimental and theoretical studies and to explore what could be done to resolve the differences.

Details about our DAC experimental gear and procedures are presented elsewhere (Akella, et al. ${ }^{1}$ ). For the structural determination at high pressures, $\mathrm{x}$-ray data were collected at the National Synchrotron Light Source beam line X-17C using an energy dispersive system.

\section{Lanthanides}

Jayaraman and Sherwood ${ }^{2}$ proposed the crystal structural sequence hcp-Sm type- dhcp-fcc for the rare-earth elements as a function of increasing pressure. Based on this, Johansson and Rosengren $^{3}$ developed a generalized theoretical phase diagram for the lanthanides. Recent experimental studies reconfirmed the structural sequence proposed by Jayaraman and Sherwood. With further increase in pressure, newer structural changes were discovered: a "six-layered hexagonal" or "distorted fcc" phase (Smith and Akella 4 and Grosshans, et al. ${ }^{5}$ ); in Pr an orthorhombic (alpha U type) phase (Smith and Akella ${ }^{6}$ and Grosshans, et al. ${ }^{7}$ ); and recently a body-centered tetragonal phase (bct) for Sm at about $90 \mathrm{GPa}$ (Vohra, et al. ${ }^{8}$ ) which is similar to that in $\mathrm{Ce}$ (Endo, et al. ${ }^{9}$ and Olsen, et al. ${ }^{10}$ ). A large volume change envisioned by theory for the post-fcc structural change, however, was not experimentally observed except for Ce and Pr. Also, 
no discernible discontinuities in $\mathrm{V} / \mathrm{V}_{\mathrm{O}}$ were noted (Fig. 1) over the various phase changes. These discrepancies between experiment and theory are noteworthy.

Phase diagram for the rare-earth metals compiled from our studies so far is presented in Fig. 2. The phase diagram is remarkably similar to the revised phase diagram proposed by Krüger, et al.11 Appearance of bct structure in Sm at about $90 \mathrm{GPa}$ (see Fig. 2) raises the question whether there is still another structural change (post bct) at higher pressure as proposed by theory. In order to answer this query, we recently finished the study of $\mathrm{Gd}$, Ho, and $\mathrm{Tb}$ to 130,186 , and $250 \mathrm{GPa}$, respectively, and the data are being analyzed. On the other hand, one of the $5 f$ metals we investigated does indeed transform to a body-centered cubic structure from an intermediate phase (see under Actinides), thus reinforcing the suggestion from theory that the ultimate high pressure phase for $f$-electron metals could be a close packed structure.

\section{Actinides:}

There are a number of theoretical studies reported in the literature to predict the crystal structural changes in the light and heavy actinides and their pressure-volume relationships. A detailed examination of all the theoretical work is not possible here. We compare our experimental results with the theoretical calculations done recently by Eriksson and Johansson from Uppsala University, J.M. Wills and others from Los Alamos National Laboratory, and Söderlind and Moriarty from Lawrence Livermore National Laboratory.

Wills and Eriksson, 12 and Eriksson, et al. ${ }^{13}$ have done calculations for $\mathrm{Th}, \mathrm{Pa}$, and $\mathrm{U}$, and Th, respectively. Söderlind, et al. ${ }^{14}$ extended these calculations to all the lighter actinides in order to develop a unified picture. These calculations were done by means of first-principles total-energy calculations using the linear 'muffin-tin' orbital method with a self-consistently calculated potential. ${ }^{15}$ The only input data for their calculation, as reported by them, are the atomic number, the crystal structure and the atomic volume.

In the case of thorium, Eriksson, et al. ${ }^{13}$ calculations could confirm the experimentally determined crystallographic phase transition from fcc $\rightarrow$ bct, albeit at a slightly high pressure 100 $\mathrm{GPa}$ versus experimental $72 \mathrm{GPa}^{16}$. For another lighter actinide $\mathrm{Np}$, the structural sequence with increasing pressure from theory ${ }^{17}$ was orthorhombic $\rightarrow(\mathrm{bct}) \rightarrow$ bcc. Calculations failed to predict a stability region for the bct neptunium phase, however experimental data showed an intermediate phase between the orthorhombic and the bcc, which has a narrow stability region and is considered to be a body-centered tetragonal structure (experiments for a positive reconfirmation are planned). The conclusion arrived at so far from experiments is that the ultimate high pressure and room temperature structure for $\mathrm{Np}$ is bcc, and this may not be the same as the bcc at low pressure and high temperature, i.e. before melting.

The predicted crystal structure sequence from theory for uranium is alpha $\mathrm{u}$ (orthorhombic) $\rightarrow$ bct $\rightarrow$ bcc. The first transformation from alpha uranium to body-centered tetragonal phase is predicted to be at $80 \mathrm{GPa}$ pressure. Experimentally in uranium no phase change could be detected even up to very high pressures at room temperature. However, the axial ratios plotted in Fig. 3 do indicate that the compressibilities of $\mathrm{a}$ and $\mathrm{b}$ axis flip around $80 \mathrm{GPa}$.

The fortuitous intersection of the $a / a o$ and $b / b o$ axial ratios at about $100 \mathrm{GPa}$ pressure is approximately the pressure at which bct structure was predicted by theory. However, unfortunately this observation does not confirm the theoretically predicted orthorhombic to bct structural phase change in uranium.

\section{Discussion:}

Even though there is general agreement between theory and experiment for the structural changes in the lanthanides and actinides, there are discrepancies still, particularly in the lighter actinides. They need to be resolved for a better agreement, so that theory can be improved. 
The discrepancy between theoretical prediction and the experimental observation regarding the structural change in uranium is noteworthy. Söderlind, et al., 18 used fixed values of $b / a$ and $c / a$ (as observed at ambient pressure) in their total energy calculations on the orthorhombic phase. Presumably, optimized $b / a$ and $c / a$ values would lower the orthorhombic total energy at high pressure, possibly eliminating the intermediate bct phase. It is also interesting to note that so far uranium is the only lighter actinide which does not go through a structural phase change at room temperature and high pressures.

If the predicted structural change in uranium is an extremely sluggish phase transformation at room temperature, possibly it could be missed experimentally. In such a case, if experiments are conducted at slightly elevated temperatures, the transformation may be accelerated. A concurrent effort to redo theoretical calculations, utilizing the experimentally determined axial and volume compression data under pressure as markers, could resolve the structural transformation discrepancy, and may also shed light on the differences in the high pressure behavior of uranium compared to other lighter actinides. We are at present focusing on that goal.

\section{Acknowledgments}

The authors would like to thank Drs. John Moriarty, Richard Grover, John Wills, Olle Eriksson, Per Söderlind, and Börje Johansson for critical discussions. Funding for this work is provided by the B-Division at Lawrence Livermore National Laboratory under DAC actinides research project. We are grateful to Drs. Bruce Goodwin and Mike Anastasio for their constant encouragement and financial support. Thanks are also due to Dr. Jing Zhu Hu of the Carnegie Institution Geophysical Laboratory for her technical help at NSLS. Work performed under the auspices of the U. S. Department of Energy by Lawrence Livermore National Laboratory under Contract W-7405-ENG-48.

\section{References:}

1. J. Akella, S.T. Weir, and B. Goodwin, Science \& Tech. Review (LLNL), March 1966.

2. A. Jayaraman and R.C. Ringwood, Phys. Rev. Lett., 112, 22 (1964).

3. B. Johansson and A. Rosengren, Phys. Rev. Lett., B-11, 2836 (1975).

4. G.S. Smith and J. Akella, Phys. Lett., 105A, 132 (1984).

5. W. A. Grosshans, Y.K. Vohra, and W.B. Holzapfel, Phys. Rev. Lett., 49, 1572 (1982).

6. $\quad$ G.S. Smith and J. Akella, J. Appl. Phys., 53, 9212 (1982).

7. W.A. Grosshans, Y.K. Vohra, and W.B. Holzapfel, J. Phys. F: Met. Phys., 13, L-147 (1983).

8. $\quad$ Y.K. Vohra, J. Akella, S. Weir, and G.S. Smith, Phys. Lett. A., 158, 89 (1991).

9. S. Endo, N. Fujioka, and H. Sasaki, High Pressure Science and Technology Vol. 1, K.D Timmerhaus and M.S. Barber, eds., Plenum Press, New York, 219 (1979).

10. J.S. Olsen, L. Gerward, V. Benedict, and J.P. Itie, Physica, 113B, 139 (1985).

11. T. Krüger, B. Merkau, W.A. Grosshans, and W.B. Holzapfel, Hig. Pr. Res., 2, 193 (1990).

12. J.M. Wills, and O. Eriksson, Phys. Rev. B. 4513879 (1992).

13. O. Eriksson, J.M. Wills, and P. Söderlind, fcc-bct phase transition in Th at extreme compressions: Theory, Los Alamos UR-92-289 (1992).

14. P. Söderlind, O. Eriksson, B. Johansson, J.M. Wills, and A.M. Boring, Nature, 374 (1995).

15. J.M. Wills, and B.R. Cooper, Phys. Rev. B, 36, 2809 (1987).

16. Y.K. Vohra and J. Akella, Phys. Rev. Lett., 67, 3563 (1991).

17. P. Söderlind, B. Johansson, and O. Eriksson, Phys. Rev. B, 52, 1631 (1995).

18. P. Söderling, Theory for crystal structures of cerium and the light actinides at low temperature and high pressure (draft). 


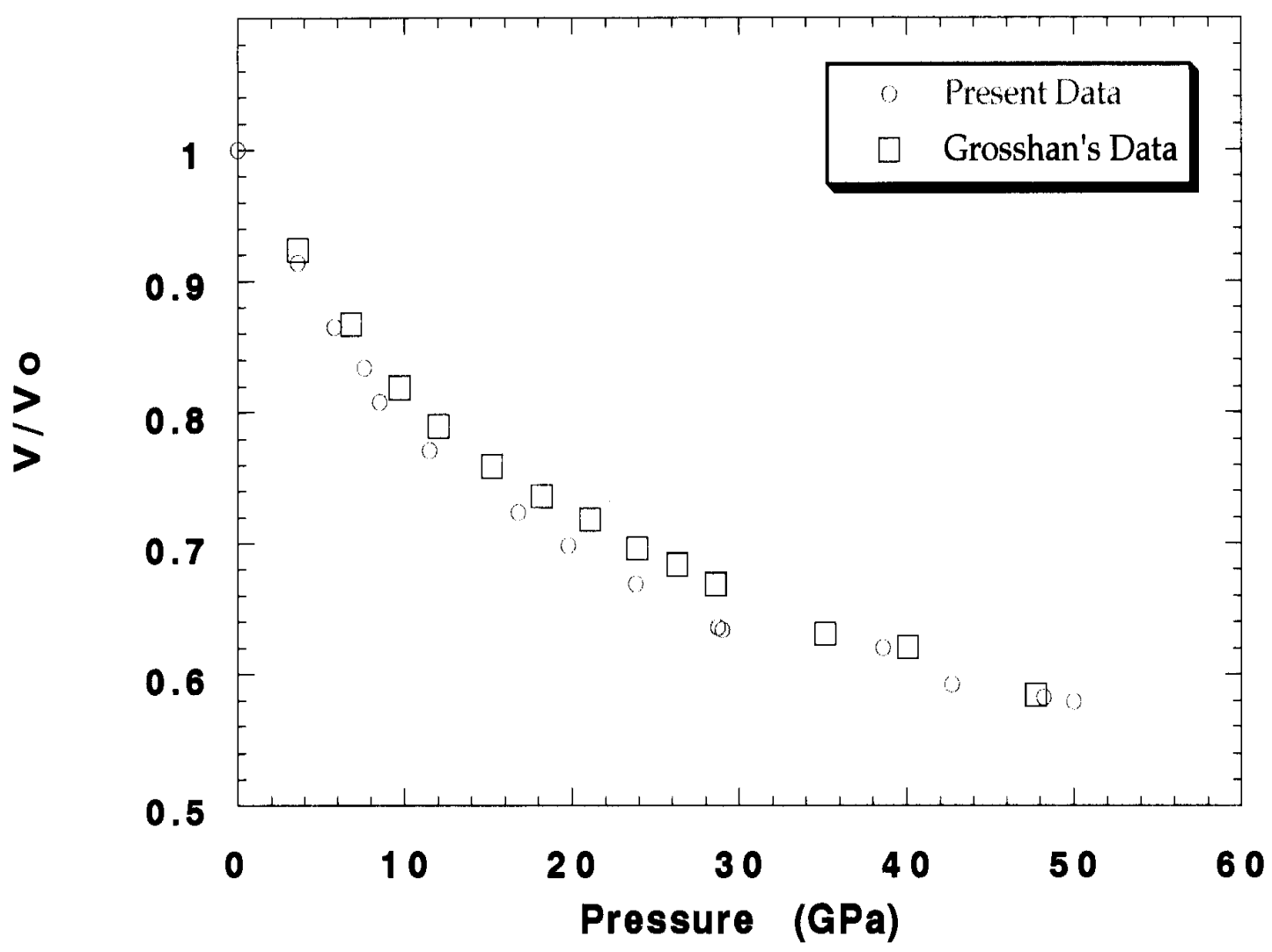

Fig. 1 Volume Compression $\mathrm{V} / \mathrm{V}_{\mathrm{o}}$ as a function of pressure for dysprosium.

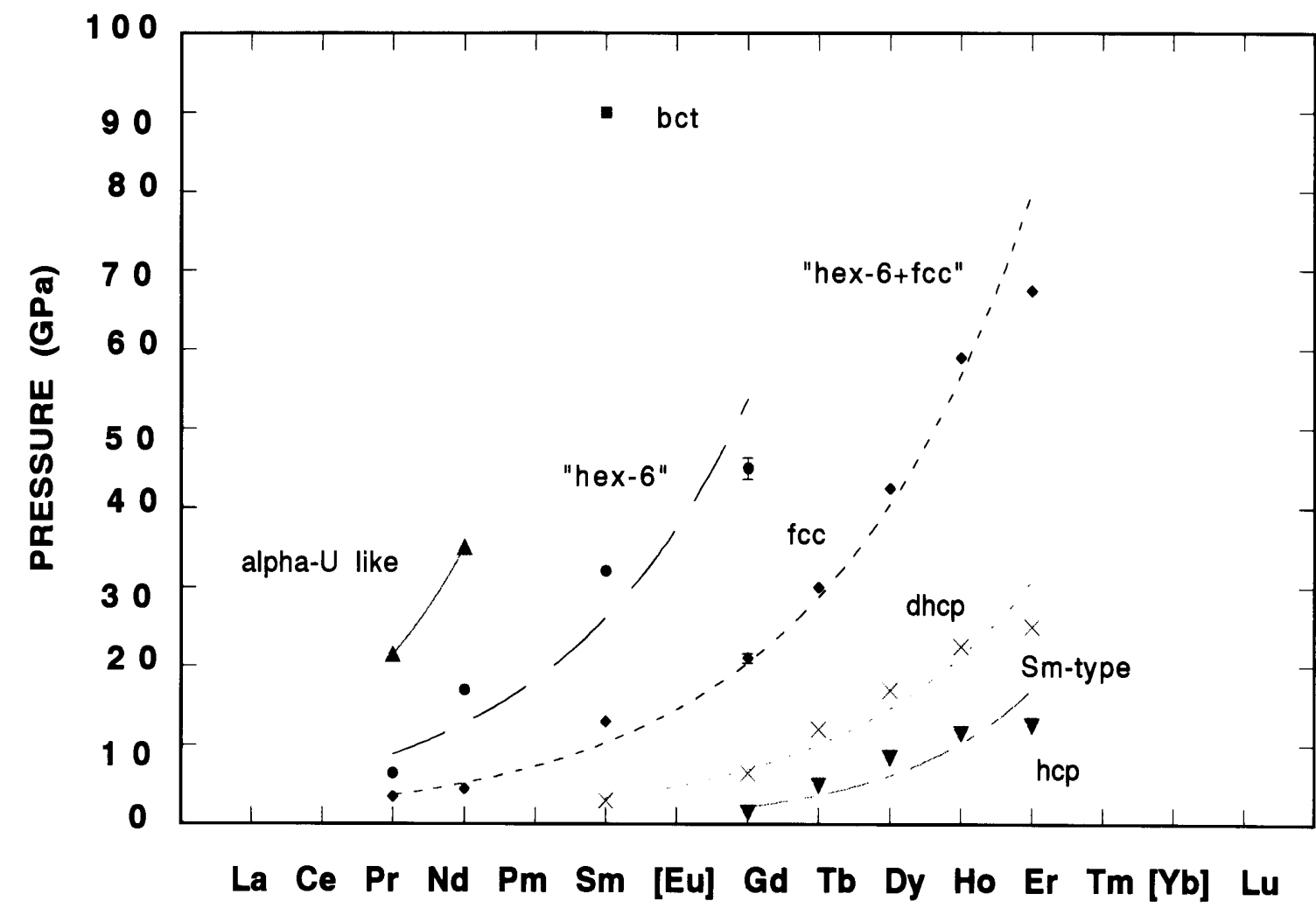

Fig. 2 Rare-earth phase diagram. 


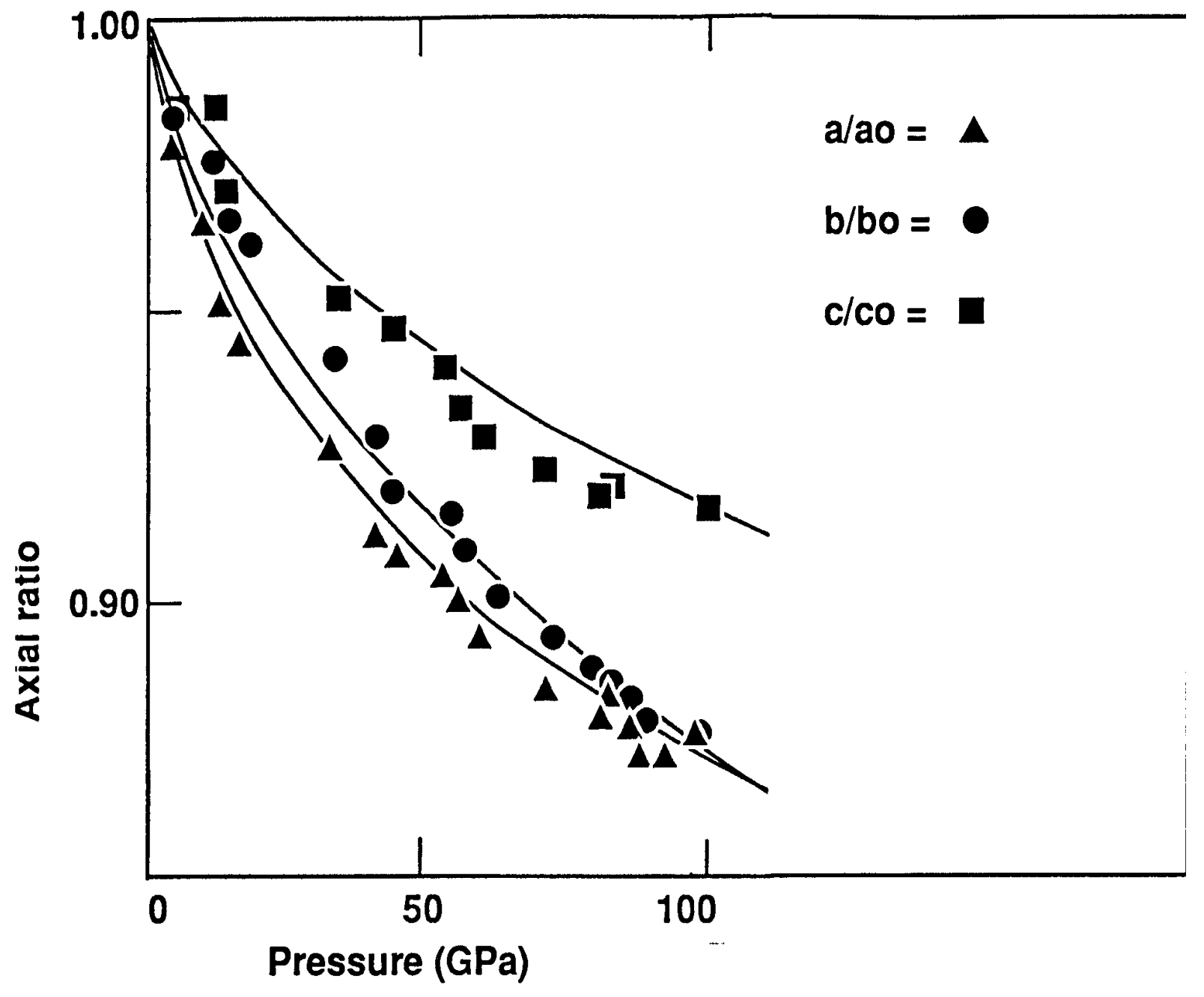

Fig. 3. The axial ratios for uranium plotted as a function of pressure. Note the change in the relative compressibilities of $a$ and $b$ axis. 


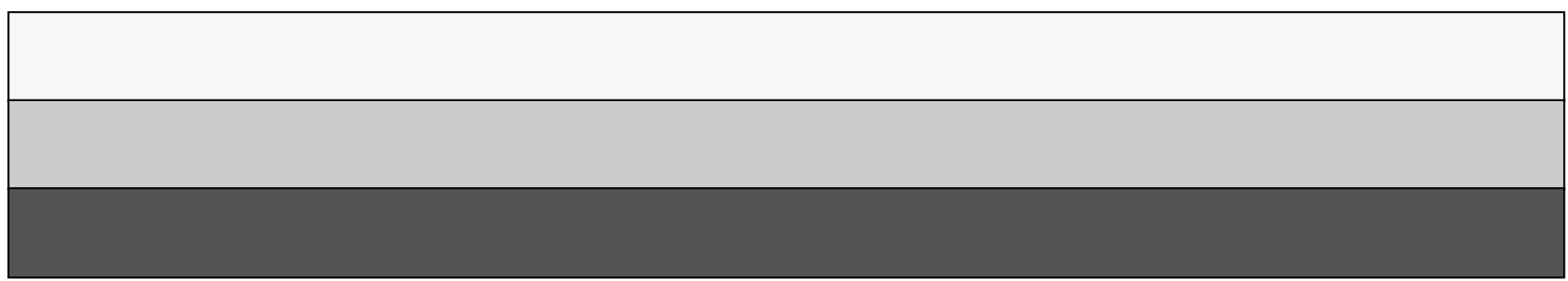

